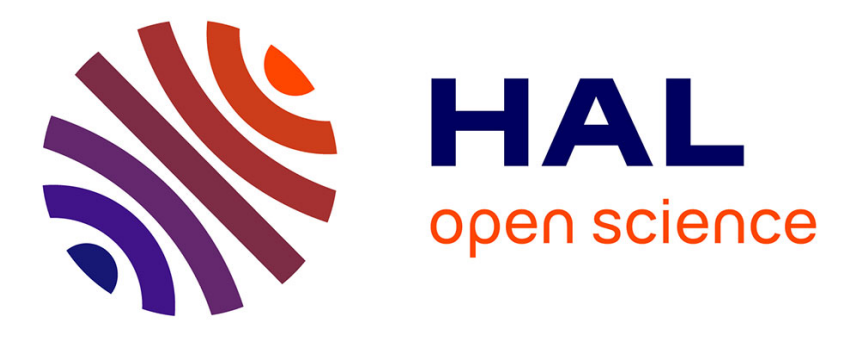

\title{
Reconstructing shaft orbit using angle measurement to detect bearing faults
}

\author{
Guillaume Bruand, Florent Chatelain, Pierre Granjon, Nadine Martin, \\ Christophe Duret
}

\section{- To cite this version:}

Guillaume Bruand, Florent Chatelain, Pierre Granjon, Nadine Martin, Christophe Duret. Reconstructing shaft orbit using angle measurement to detect bearing faults. Mechanical Systems and Signal Processing, 2020, 139, pp.106561. 10.1016/j.ymssp.2019.106561 . hal-02408146

\section{HAL Id: hal-02408146 \\ https://hal.science/hal-02408146}

Submitted on 12 Dec 2019

HAL is a multi-disciplinary open access archive for the deposit and dissemination of scientific research documents, whether they are published or not. The documents may come from teaching and research institutions in France or abroad, or from public or private research centers.
L'archive ouverte pluridisciplinaire HAL, est destinée au dépôt et à la diffusion de documents scientifiques de niveau recherche, publiés ou non, émanant des établissements d'enseignement et de recherche français ou étrangers, des laboratoires publics ou privés. 
1

2

3

\title{
Reconstructing shaft orbit using angle measurement to detect bearing faults
}

\author{
Guillaume Bruand $^{\mathrm{a}, \mathrm{b}, *}$, Florent Chatelain ${ }^{\mathrm{a}}$, Pierre Granjon ${ }^{\mathrm{a}}$, Nadine \\ Martin $^{\mathrm{a}}$, Christophe Duret ${ }^{\mathrm{b}}$ \\ ${ }^{a}$ Univ. Grenoble Alpes, CNRS, Grenoble INP ${ }^{1}$, GIPSA-lab, 38000 Grenoble, France \\ ${ }^{b} N T N-S N R$ Roulements, 1 Rue des Usines, 74000 Annecy, France
}

\begin{abstract}
The use of Instantaneous Angular Speed (IAS) in condition monitoring of rotating machines is an appealing alternative to traditional approaches such as those based on accelerometers: the direct angular sampling characteristic of IAS measurements has proven to be a favoured framework to study mechanical phenomena involved in rotating machines. Physical models have been established to link the variations of IAS to torque disturbances in case of mechanical fault such as bearing failure. In this paper an original point of view is given on the IAS measurement by linking IAS variations and shaft vibrations. First the orbit of a rotating shaft is studied to show that it contains useful information about the health state of the bearing. Then angle position sensors traditionally used in IAS measurements are combined to reconstruct this orbit. It is shown that IAS measurements are sensitive to the shaft vibrations, which may be used advantageously to enhance the diagnosis possibilities. Finally the links between various defect sizes and the reconstructed orbits are investigated to propose a fault severity indicator.
\end{abstract}

Keywords:

Instantaneous Angular Speed, Bearing fault, Condition Monitoring, Orbit, Fault severity

\footnotetext{
${ }^{*}$ Corresponding author

Email address: guillaume.bruand@grenoble-inp.fr (Guillaume Bruand)

${ }^{1}$ Institute of Engineering Univ. Grenoble Alpes
} 


\section{Introduction}

Condition monitoring is a common approach to reduce costs in industrial environments. Indeed, an early diagnosis of an incoming failure can prevent a machine from serious damages, and allows the customer to plan maintenance operations to minimize the associated costs. In the field of rotating machines, bearings are a key component prone to failure, and a correct diagnosis can be challenging for the analyst. Various measurement techniques have been developed for bearing condition monitoring, such as vibrations [1, 2], acoustic emissions [3], or oil analysis [4]. More recently, the Instantaneous Angular Speed (IAS) has been used effectively in the condition monitoring scope $[5,6,7]$. The bearing failure case itself has been studied by Renaudin et al. [8].

The IAS is a measurement based on the angular position of a rotating machine. The principle is to associate a sensor with a target linked to the rotating part to get a high resolution angle measurement, the rotating speed being computed with the help of a high frequency clock. The sensor may be either optical, associated with a zebra-tape target, or magnetic, such as Hall cell arrays or magnetoresistances, associated with an "encoder" which is an alternation of North and South magnetic poles.

At each discrete angular position, the sensor generates pulses which are processed to obtain the angle of the rotating part. The measurement of IAS itself has been the object of several studies. Li et al. [9] presented two methods to estimate the IAS, one based on counting the number of pulses at the output of the sensor in a given time, and the other one counting the elapsed time between two consecutive pulses. Badaoui and Bonnardot [10] showed that, using a counting system, angular sampling is a non-uniform time sampling introducing a noise which power is proportional to the jitter variance. André et al. [11] studied the accuracy of the counting system used to estimate the IAS, and Leclere et al. [12] made a deeper analysis of the measurement errors and their influence on the speed estimation, taking the irregularity of the magnetic coder and the jitter of the clock into account. Palermo et al. [13] proposed a calibration tool to minimize the geometrical error when estimating the angular speed with zebra-tapes.

The most interesting feature of IAS is that it is naturally linked to the angular phenomena involved in rotating machines, making it relevant for their analysis; it has then attracted attention to a new field of research focused on the angular point of view provided by the IAS measurement. 
A first attempt to model the IAS variations induced by a defect (gear, bearing) has been made by Bourdon et al. [14] where a numerical model for an angular periodic disturbance is proposed. This approach has been validated later [15] where an experimental study confirms the interest of the method in non-stationary operating conditions. A link between IAS alteration and torque variations due to a bearing fault has been proposed by Gomez et al. [16]. This latter work may be the only existing work trying to give a physical explanation to the observed IAS variations in presence of a bearing fault. The hypothesis is that rolling elements are creating a resisting torque due to their rolling resistance modelled by Gomez as proportional to the normal contact forces. Hence a flaking in one of the bearing races induces a disturbance of these contact forces, leading to torque variations, and thus to slight rotation speed modification, measured by IAS.

In this paper, a new point of view is given on the physical model underlying IAS variations in presence of a bearing fault. The proposed approach deduces torque variations directly from the contact forces inside a faulty bearing, as an alternative to the rolling resistance model proposed by Gomez et al. [16]. Furthermore, it is shown that a bearing fault induces displacements of the rotating shaft, which may be measured as slight disturbances of the trajectory. A new theoretical model shows that, quite surprisingly, angle measurement sensors are sensitive to these displacements, which has not been taken into account in the traditionnal IAS measurements yet. As a consequence, the way of measuring IAS is rethought at the light of these new developments, and an original measurement apparatus combining several sensors is proposed.

This paper is organized as follows. In the second section, a new mechanical model is proposed to show that the orbit of a rotating shaft carries on information about a bearing fault.

In the third section, it is proven that such orbits, and the information they provide, can be indirectly retrieved thanks to several angle measurement sensors placed on the same shaft. Mathematical material is provided along with numerical simulation to support this statement.

In the fourth section, to validate the proposed approach, an experiment is conducted using eddy-current displacement sensors as proximity probes, and Anisotropic Magnetic Resistances as angle measurement sensors. Results obtained by both kind of sensors are then compared to confirm the hypothesis made in this paper. Several faulty bearings have been used in various conditions, and a simple indicator of the fault severity is derived from the shaft 
orbits.

Finally some conclusions and perspectives are drawn in the last section.

\section{Mechanical model of a rotating beam with faulty bearing}

In this first part, a new mechanical model is proposed to describe the contact forces inside a ball bearing. Two goals are pursued here:

1. The first one is to propose a novel explanation to the torque variations induced by a bearing fault, which have been described in [16] as directly linked to the rolling resistance of rolling elements.

2. The second objective is to completely describe the orbit of a rotating shaft in the radial plane, and to prove that relevant information about the health state of the bearing may be retrieved.

An abundant literature exists on the bearing fault model, which have been the basis of vibratory analysis. The localized defect case, which is of prime interest in the fatigue failure scope, has been the subject of increasing attention. A first model has been proposed by Tandon and Choudhury [17], showing that shocks occur when rolling elements pass through a localized defect and are modulated by the load effect. It has been improved later by Sopanen and Mikkola [18] taking distributed defects such as waviness into account, and further by Sawalhi and Randall [19] who included slippage in the model.

The aim of the present work is not to give a comprehensive description of every complex phenomena involved in a bearing fault, but to introduce some thoughts about the shaft behaviour and the torque variations. Hence several hypothesis and simplifications have been made in order to carry on the simulation:

1. Cage deflection is neglected such that rolling elements stay at the same relative angular positions. The cage does not slip during its rotation.

2. There are no centrifugal forces acting on the rolling elements.

3. Temperature influence is neglected, as well as elasto-hydrodynamic effects.

4. The waviness of the raceways has not been taken into account, however the proposed approach may be used for this purpose.

In recent years, extensive research has been done to study more and more sophisticated defect models [20]. New developments are now focused on the 
estimation of the fault severity to predict the Remaining Useful Life of a faulty bearing. In model based approaches the defect size is estimated, as proposed by Sawalhi and Randall [21] who retrieves information coming from the impacts of rolling elements at both entry and exit of the defective zone. In [22] the traditional Hertzian theory of contact is modified inside the defect zone to model the behaviour of rolling elements with better accuracy. Another illustration can be found in [23] where the cage and traction dynamics are taken into account inside an advanced multi-body model. The approach presented here, while being simpler and quite intuitive, has the advantage of being able to show how a potential bearing defect may lead to IAS disturbances. In addition, the link with the shaft displacement is explored for the first time.

\subsection{State space equations}

First, a simple 6-degrees of freedom beam has been simulated using a Timoshenko model with finite element analysis (see Fig.1). The 6 degrees of freedom are the 3 displacements $\left(\begin{array}{lll}x & y & z\end{array}\right)$ and 3 rotations $\left(\begin{array}{lll}\theta_{x} & \theta_{y} & \theta_{z}\end{array}\right)$ along every axes. The shaft axis is along $z$, associated with its rotation $\theta_{z}$, while $x$ and $y$ axes define the radial plane.

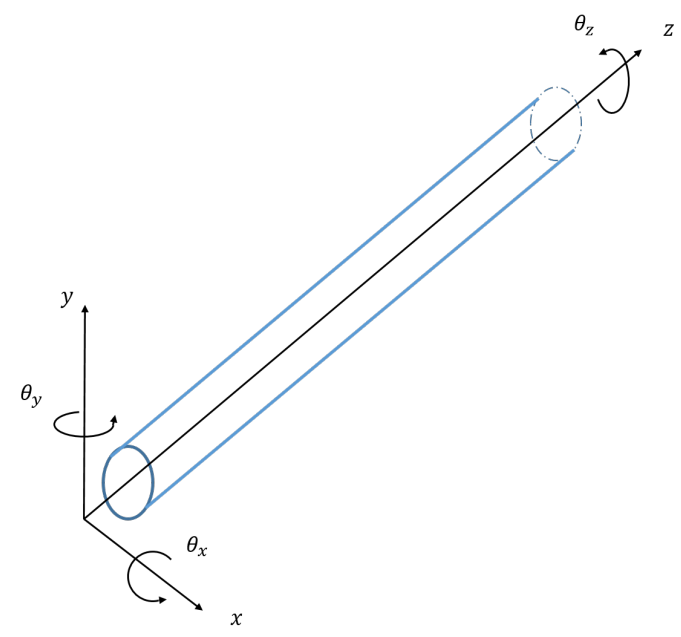

Figure 1: Coordinates system of the 6 -dof beam model with the 3 displacements $x, y, z$ and 3 rotations $\theta_{x}, \theta_{y}, \theta_{z}$

The beam is discretized into $P-1$ finite elements linked by $P$ nodes. Denote $X_{i}$ the vector of displacements for the node $i$, i.e. $X_{i}=\left(x_{i} y_{i} z_{i} \theta_{x, i} \theta_{y, i} \theta_{z, i}\right)^{T}$ 
and $V_{i}=\dot{X}_{i}$ its time derivative. The global vector of displacement $X=$ $\left(X_{1} X_{2} \ldots X_{P}\right)^{T}$ and the global vector of velocity $V=\left(\dot{X}_{1} \dot{X}_{2} \ldots \dot{X}_{P}\right)^{T}$ are built. The system is described by the governing equations of motion

$$
\begin{aligned}
& \frac{\mathrm{d} X(t)}{\mathrm{d} t}=V(t) \\
& M \frac{\mathrm{d} V(t)}{\mathrm{d} t}+C V(t)+K X(t)=F_{\text {ext }}
\end{aligned}
$$

where mass matrix $M$, damping matrix $C$ and stiffness matrix $K$ are built in the scope of the beam theory using mass $m=30 \mathrm{~kg}$, diameter $d=75.9$ $\mathrm{mm}$, Young modulus $E=210 \mathrm{GPa}$, and Poisson ratio $\nu=0.3$. These numerical values have all been chosen to match those of the experimental bench described in section 4 . Details are given in $[24,25]$ from which these matrices have been adapted. The vector $F_{\text {ext }}$ represents the external forces and torques applied to the system. Radial load in the $(x y)$ plane subjects the beam to bending. On the other hand, axial load (along the $z$ axis) acts in traction/compression on the beam. The motor torque will be transmitted along $z$.

In the following section the external forces $F_{\text {ext }}$ are computed.

\subsection{Bearing deflection and contact forces}

At each time step, the external forces applying on the beam are computed, and especially the contact stresses at the bearing locations. These forces have been modelled by Hertzian contact forces caused by the deflection of the rolling elements when the shaft moves from its original position (see Fig.2).

To take the geometry of an angular contact bearing into account, it is convenient to use spherical coordinates to give the position of the rolling elements. A set of local coordinate system $\left(e_{r}^{j} ; e_{\theta}^{j} ; e_{\varphi}^{j}\right)$ described in Fig.3 has been obtained by rotating the initial coordinates system in the direction of the $j^{\text {th }}$ rolling element. The angles $\theta_{j} \in[0,2 \pi$ [ define the angular position in the radial plane $\left(e_{x} ; e_{y}\right)$ and $\varphi_{j} \in\left[-\frac{\pi}{2}, \frac{\pi}{2}\right]$ the angular contact.

The matrix

$$
P_{j}=\left(\begin{array}{ccc}
\cos \varphi_{j} \cos \theta_{j} & \cos \varphi_{j} \sin \theta_{j} & \sin \varphi_{j} \\
-\sin \theta_{j} & \cos \theta_{j} & 0 \\
-\sin \varphi_{j} \cos \theta_{j} & -\sin \varphi_{j} \sin \theta_{j} & \cos \varphi_{j}
\end{array}\right)
$$


Figure 2: When moving from its original position, the inner ring (IR) induces deflection on the $j^{\text {th }}$ rolling element pressed against the fixed outer ring (OR) located at the angle $\theta_{j}$. The contact force $F_{j}$ is proportional to the total deflection obtained by projecting the displacement $\overrightarrow{O O^{\prime}}$ in the radial direction $\overrightarrow{O M}, M$ being the contact point between the race and the rolling element.

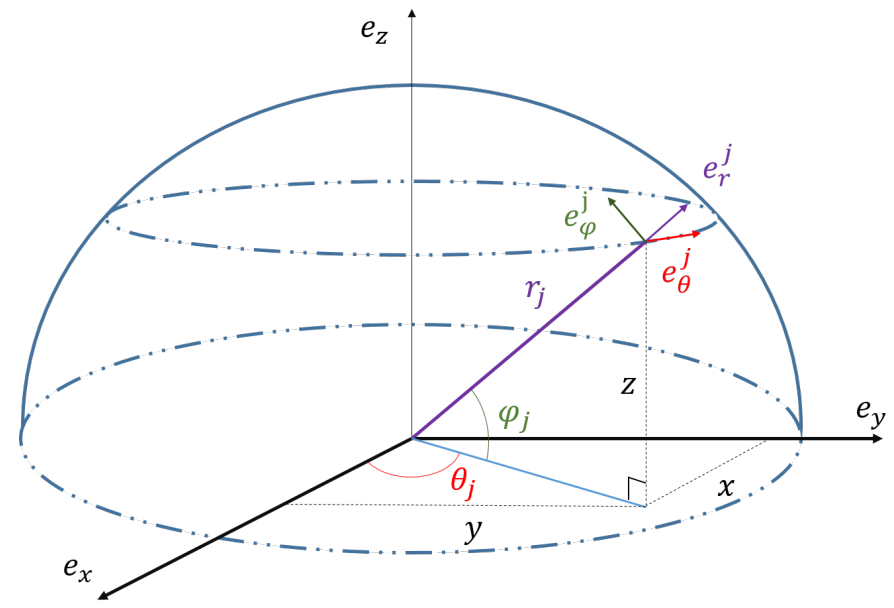

Figure 3: The local coordinates system $\left(e_{r}^{j}, e_{\theta}^{j}, e_{\varphi}^{j}\right)$ linked to the $j^{\text {th }}$ rolling element. 
is the rotation matrix of the transformation from the initial coordinates of the shaft $U_{0}$ to the new coordinates $U_{j}$ in the local coordinate system linked to the $j^{\text {th }}$ rolling element, such as

$$
U_{j}=P_{j} U_{0}
$$

When the shaft moves from its original position, the rolling elements are subjected to a load leading to deflection, which is the relative displacement between the two rings of the bearing minus the clearance (see Fig.2). The total deflection of the system \{inner ring + outer ring + rolling element $j$ \} noted $\delta_{j}$ is simply expressed as the projection of the shaft displacement in the $j^{\text {th }}$ radial direction

$$
\delta_{j}=U_{j} \cdot e_{r}^{j}-c\left(\theta_{j}\right)
$$

where $c$ is the bearing inner clearance. If $r_{j}$ is the radial component of the displacement $U_{j}$, the deflection is then written

$$
\delta_{j}=r_{j}-c\left(\theta_{j}\right) .
$$

In the Hertzian theory of contact, the bearing normal contact force is directly proportional to the deflection of the different elements. It is given by

$$
F_{j}=k_{b} \max \left\{0, \delta_{j}\right\}^{\alpha} e_{r}^{j}
$$

where $F_{j}$ is the effort on the $j^{\text {th }}$ rolling element, $k_{b}$ is the equivalent stiffness of the set $\{$ inner ring + outer ring + rolling element $\}$ and $\alpha$ is a coefficient depending on the type of rolling elements used (balls, rollers). The equivalent contact stiffness $k_{b}$ can be calculated in terms of the geometrical parameters of the bearing, and in the case of ball bearings $\alpha=3 / 2[26]$.

When there is no deformation (i.e. when the relative displacement of the rings is lower than the clearance, or $\left.\delta_{j} \leq 0\right)$, the load applied on the corresponding element is null. The global force acting on the shaft is obtained by summing the contributions of each rolling element

$$
F_{\text {bearing }}=-\sum_{j} F_{j}=-k_{b} \sum_{j} \max \left\{0, \delta_{j}\right\}^{\alpha} e_{r}^{j} .
$$

The total external forces $F_{\text {ext }}$ is obtained by summing the contributions of external forces (torque and loads), and inner bearing contact forces $F_{\text {bearing }}$. 


\subsection{Modelling a bearing fault}

Two approaches exist in the literature to model a bearing fault. In the first one, a defect on one of the raceways is introduced by adding a clearance in the damaged zone; hence the defect is modelled as a hole in the ring with a given shape, as proposed by Sawalhi and Randall [19]. The second approach uses finite element analysis to describe the contact forces and hence the deflection of the rolling element when passing through the defect zone; an example is given in [22] where an equivalent stiffness is computed for different types of defects, depending on the relative sizes of the defect and the rolling element. In the present paper, an intermediate path is adopted: the clearance approach is retained and the contact stiffness described in Eq.(6) is kept constant, but the contact forces are derived in an original way, using the defect profile and finite element analysis. This approach has the advantage to remain simple while explaining the contact forces at the origin of the shaft displacement.

For a fault located at angle $\theta_{d}$ of width $2 a$ (oriented in the raceway direction), the clearance is obtained by summing the bearing clearance $c_{\text {bearing }}$ and the defect linked clearance $c_{d}$,

$$
c\left(\theta_{j}\right)=c_{\text {bearing }}+c_{d}\left(\theta_{j}\right)
$$

where

$$
c_{d}\left(\theta_{j}\right)= \begin{cases}f\left(\theta_{j}\right) & \text { if } \theta_{j} \in\left[\theta_{d}-a ; \theta_{d}+a\right] \\ 0 & \text { elsewhere }\end{cases}
$$

and $f$ is some arbitrary shape function.

The defect is modelled as a hole in one of the rings (Fig.4a), corresponding to a rectangular window function $f$. The contact force has been assumed to be radial in Eq.(6), i.e. along $e_{r}^{j}$, which is reasonable without defect but does not hold when a rolling element passes through a flaking zone. In this section, this model is enhanced by considering a more complex defect shape, with a force being no longer radial (Fig.4b).

The direction of the $j^{t h}$ rolling element normal contact force $F_{j}$ is directly deduced from the shape of the defect, so that the force is always directed along the normal to the raceway surface. More precisely, when $\mathfrak{R}=\left(e_{r}^{j}, e_{\theta}^{j}, e_{\varphi}^{j}\right)$ is the local set of coordinates, and $c(\theta)$ the shape of the defect, the normal component is 


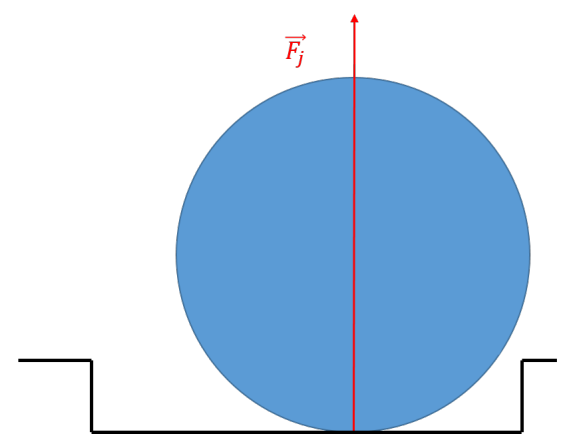

(a) Simple clearance model.

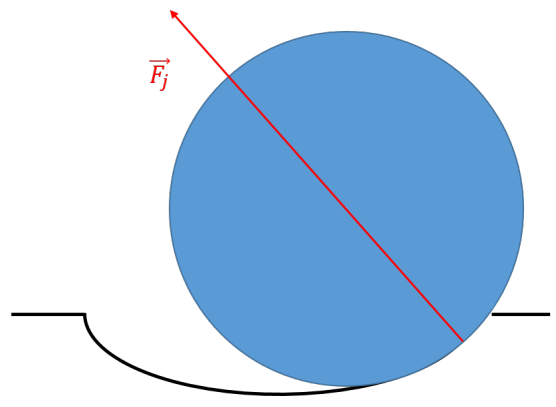

(b) Advanced model.

Figure 4: Contact forces in flaking zone - scale is not respected.

$$
F_{j}=-\left\|F_{j}\right\|\left(1+\dot{c}(\theta)^{2}\right)^{-1 / 2}\left(e_{r}^{j}+\dot{c}(\theta) e_{\theta}^{j}\right)
$$

where $\left\|F_{j}\right\|=k_{b} \max \left\{0, \delta_{j}\right\}^{\alpha}$ as given in Eq.(6). Proof is given in appendix.

This model explains the variations of external forces introduced by a defect as a modification of the normal contact forces on each of the rolling elements when they go through the defective area. Furthermore, this approach leads to torque disturbances that are directly linked to the rotation speed and the associated IAS measurement. These disturbances are discussed in the next section.

\subsection{Torque disturbances}

When the contact forces remain normal inside the bearing, there is no resultant torque and thus no speed variations even in presence of a defect. To explain the origin of IAS variations observed in measurements, Gomez et al. [16] introduced a rolling resistance effect, from which a tangential component directly proportional to the normal component is added to the contact force. This tangential force is obtained with an arbitrary rolling resistance coefficient, and leads to a resulting torque.

To avoid this arbitrariness, the presented approach deduces the value of the tangential component from the race profile in Eq.(9). Furthermore, the clearance model of Eq.(8) is used to model the waviness of the race as it is done with a localized defect.

\subsection{Simulation results}

Once external forces and model of Eq.(1) have been set up, the Matlab Ode 45 solver is used to solve the system of differential equations, using an 
explicit adaptive Runge-Kutta method [27].

The simulated bench is designed to be as close as possible to the experimental one used in the next section. The shaft is subjected to both axial and radial forces, respectively equal to $F_{a}=4 k N$ and $F_{r}=8.8 k N$. The shaft is supported by two main bearings (ref. SNR 22212EAW33). At both extremities of the shaft, external forces are applied on the test bearings, which are double row ball bearings (ref. SNR XTGB42000). An outline of the bench is given in Fig.5.

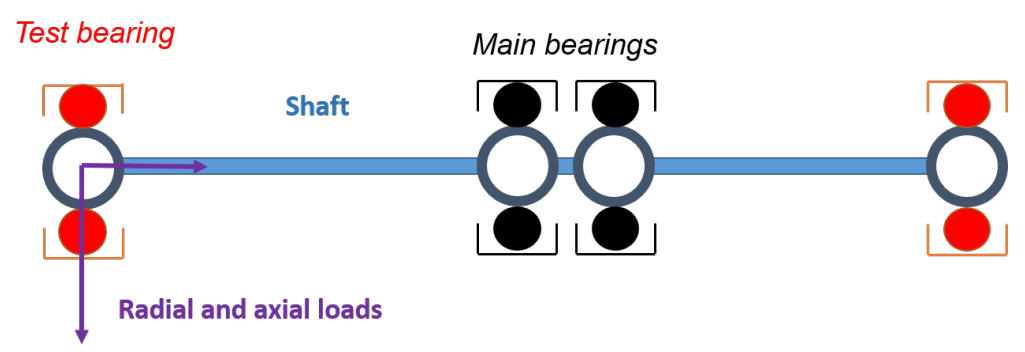

Figure 5: Outline of the experimental test bench.

The shaft is in steel of density $7850 \mathrm{~kg} \cdot \mathrm{m}^{-3}$, of length $0.76 \mathrm{~m}$ and diameter $8 \mathrm{~cm}$. The test bearing characteristics are summarized in Table 1.

\begin{tabular}{|l|c|c|}
\hline Bearing & Test Bearing & Main Bearing \\
& SNR XTGB42000 & SNR 22212EAW33 \\
\hline Type & Angular ball bearing & Roller bearing \\
Nb of rolling elements & 11 & 18 \\
Inner ring diameter & $38.42 \mathrm{~mm}$ & $60 \mathrm{~mm}$ \\
Outer ring diameter & $65.64 \mathrm{~mm}$ & $110 \mathrm{~mm}$ \\
Cage diameter & $52 \mathrm{~mm}$ & $85 \mathrm{~mm}$ \\
Rolling element diameter & $13.49 \mathrm{~mm}$ & $18.7 \mathrm{~mm}$ \\
Rolling element length & nd & $11.2 \mathrm{~mm}$ \\
Internal clearance & $20 \mu \mathrm{m}$ & $50 \mu \mathrm{m}$ \\
Contact angle & $36^{\circ}$ & $0^{\circ}$ \\
\hline
\end{tabular}

Table 1: Bearings characteristics.

A total of 10 nodes has been considered to discretize the shaft model. The rotating speed is set up to $500 \mathrm{rpm}$. A rectangular window of various widths and depths have been generated to model a hole in one of the raceways and 
study the shaft behaviour in several different fault cases (see Fig.6). Edges of the defect area have been smoothed to avoid discontinuities. Results are given for window widths of $1 \mathrm{~mm}, 2 \mathrm{~mm}, 3 \mathrm{~mm}$, and depths of $10 \mu \mathrm{m}, 50 \mu \mathrm{m}$ and $200 \mu \mathrm{m}$. The defect is simulated on the inner (non-rotating) ring of one test bearing, the entry of the defect being at the same angular position for each case study.

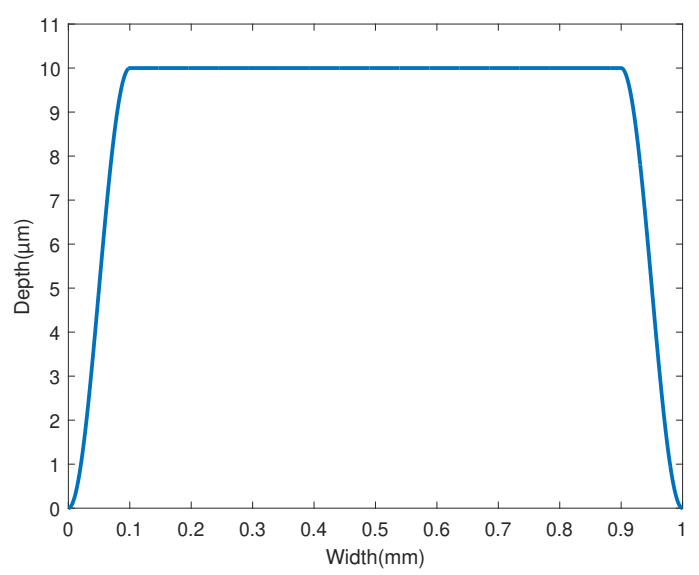

Figure 6: A rectangular window is used to model the defect, the hypothesis being that a fatigue crack leads to a large removal of matter when reaching the surface, forming a hole in the raceway.

Fig.7 shows that the nature of the displacement induced by a localized fault depends on both depth and width of the defect. The orbit drawn Fig. 7b shows that the shaft displacement is mainly vertical, i.e. in the defect direction. It can be decomposed in two parts each time a rolling element passes through the hole in the ring:

- at the entry of the defect, the rolling element is unloaded as the clearance sharply increases, so that the shaft moves towards this direction

- when the rolling element reaches the exit zone, the clearance sharply decreases and a shock happens as the ball is loaded again.

In order to validate these assumptions, two other shapes with a smoothed entry (Fig.8a) or a smoothed exit (Fig.8b) have been simulated. It appears (Fig.9) that the shock is effectively due to the sharp load/unload of one of the rolling elements. Indeed with a smooth entry (or exit) the rolling element follows the profile of the defect and no shock occurs. 

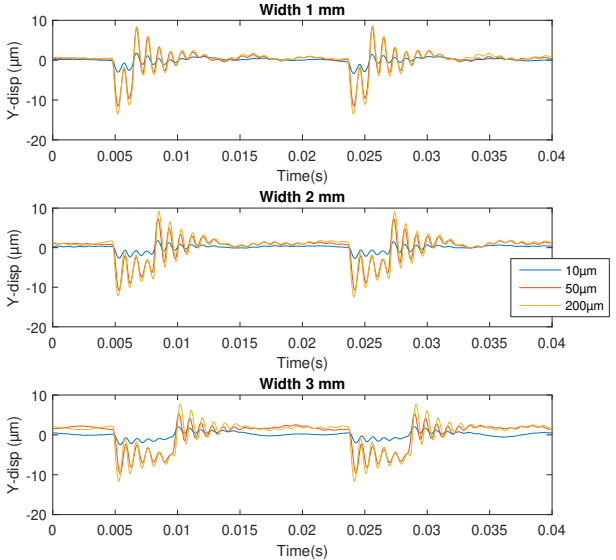

(a) Vertical displacements obtained for different defect sizes. The displacement amplitude increases with depth, while two shocks are observed at entry and exit of the defect.
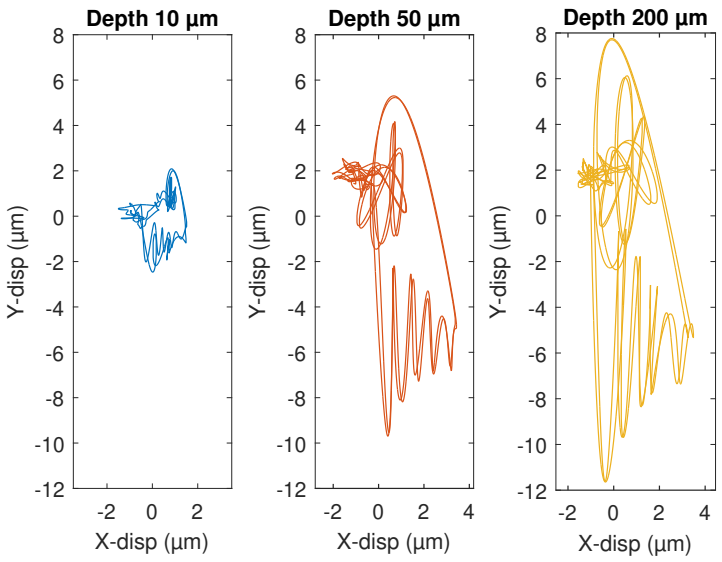

(b) Corresponding orbits for the $3 \mathrm{~mm}$ width case. Vertical displacement is plotted versus horizontal displacement.

Figure 7: Results obtained for a rectangular defect shape. Colours have been used to compare the influence of defect size.

Simulations have been done with several speed conditions (Fig.10) corresponding to experimental tests. It can be observed that the rotation speed has little influence on the shaft behaviour.

\subsection{Insights provided by the mechanical model}

The proposed mechanical model shows that a bearing fault induces shaft displacements viewed as disturbances of the trajectory. More precisely, the sudden clearance increase occurring when a rolling element goes through a localized defect leads to a displacement of the shaft in this direction, along with a shock when the rolling element is loaded again. Using an original approach to model contact forces in an arbitrary shaped defect, the shaft orbit in a radial plane has been studied with various fault cases. A first approach indicates that both depth and width of the fault influence the amount of shaft displacement. The following section shows in what extent angle measurement sensors are sensitive to these displacements, proving that IAS speed signals contain information directly linked to the vibrations of the shaft. As a result, a new approach is proposed to enhance IAS measurements by retrieving optimally the information about a potential bearing fault contained in the orbit. 


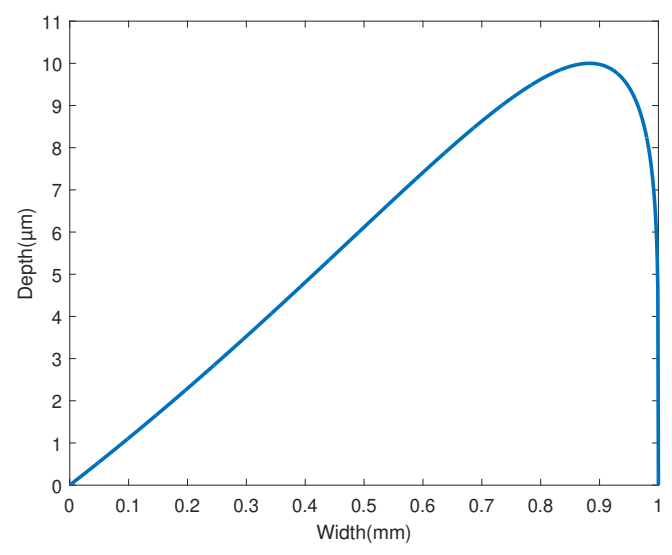

(a) Smoothed entry.

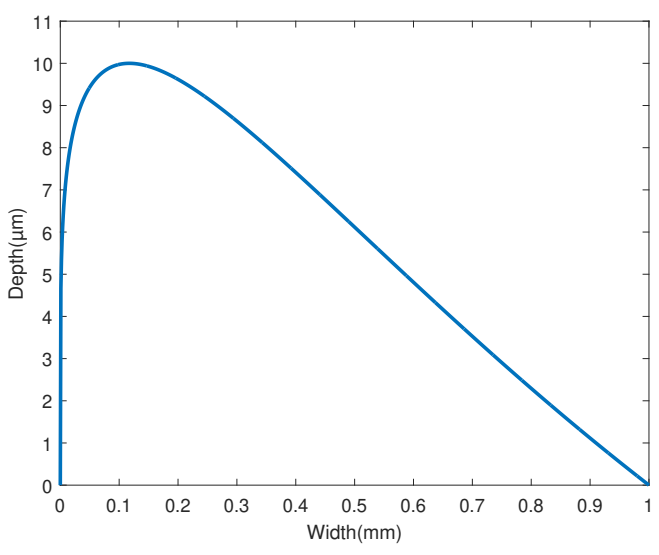

(b) Smoothed exit.

Figure 8: Asymmetric defect shape used to model a smooth entry/exit of the defect.
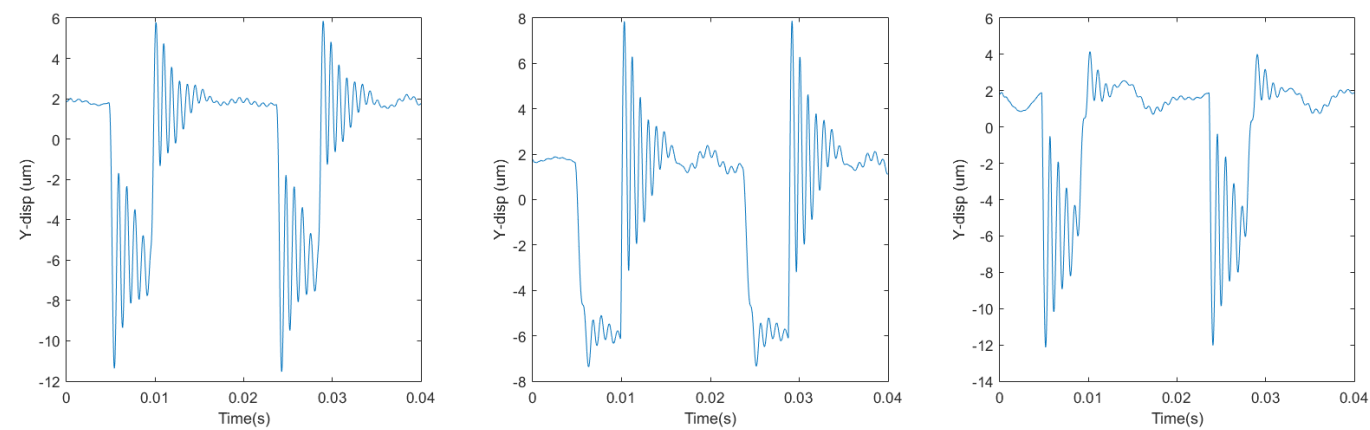

Figure 9: Vertical displacement for 3 different defect profiles : at left the rectangular shaped defect, at centre and at right the 2 asymmetric shaped defects. 

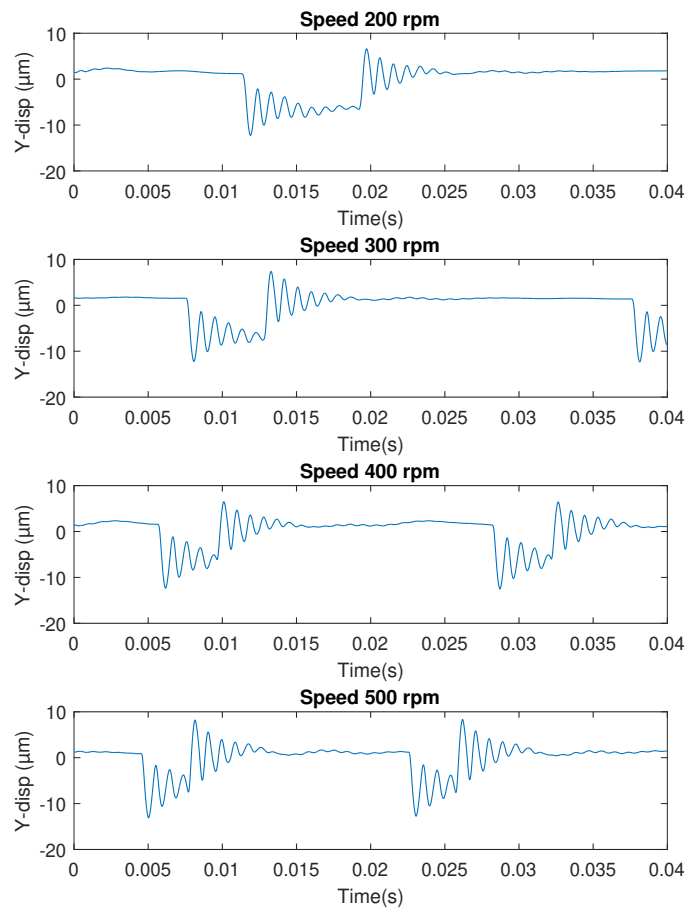

Figure 10: Vertical displacements obtained for different speeds. The simulated defect is $200 \mu \mathrm{m}$ deep and $2 \mathrm{~mm}$ wide. Shocks are closer when rotation speed increases but it has no clear effect on the shock amplitude. 


\section{A theoretical approach to reconstruct orbits using angle mea- surement}

IAS is computed from high resolution angle measurements, provided by magnetic or optical probes. The sensors are fixed to the machine, while a target is rotating with the shaft and gives the angular reference. The traditional point of view regarding IAS is that a bearing fault induces rotation speed disturbances, that may be observable in the signals. It is shown in this section that angle measurement sensors are sensitive to shaft displacements, which can be explained by the target moving before the probe. Furthermore, the shaft trajectory can be retrieved using several angle measurement sensors, which can be used advantageously to diagnose bearings, as suggested by the mechanical model studied in the preceding section. The demonstration begins with some geometry.

\subsection{Mathematical model}

An orthonormal coordinate system $\mathcal{R}=\left(O, \mathbf{e}_{\mathbf{x}}, \mathbf{e}_{\mathbf{y}}\right)$ is considered, where $O$ is the shaft centre at its initial position $(t=0)$. A sensor is placed in $A$ at a distance $R$ from $O$, and at an angle $\theta$, such that $\overrightarrow{O A}=R\left(\cos (\theta) \mathbf{e}_{\mathbf{x}}+\sin (\theta) \mathbf{e}_{\mathbf{y}}\right)$. For some $t>0$, the shaft has moved from its original position by a distance $Z$ in the direction $\varphi$, such that the centre of the shaft is now $O^{*}$ with $\overrightarrow{O O^{*}}=Z\left(\cos (\varphi) \mathbf{e}_{\mathbf{x}}+\sin (\varphi) \mathbf{e}_{\mathbf{y}}\right)$, forming a new system $\mathcal{R}^{*}=\left(O^{*}, \mathbf{e}_{\mathbf{x}}, \mathbf{e}_{\mathbf{y}}\right)$ as depicted in Fig.11.

The angular position of the sensor in $\mathcal{R}^{*}$ is noted $\theta^{*}$, and is given from the original angle $\theta$; from Fig.11 it can be deduced that

$$
\tan \left(\theta-\theta^{*}\right)=\frac{\tilde{y}}{R-\tilde{x}}
$$

where $\tilde{x}=Z \cos (\varphi-\theta)$ and $\tilde{y}=Z \sin (\varphi-\theta)$ are the displacements of the shaft in the direction of the sensor and in the normal direction to the sensor respectively.

The new angle is now

$$
\theta^{*}=\theta-\arctan \left(\frac{\tilde{y}}{R-\tilde{x}}\right) .
$$

In case of small displacements, that is $Z \ll R$, a first order approximation gives 
Figure 11: Illustration of the shaft displacements: the shaft centre moves from its original position $O$ to the new position $O^{*}$ such that the angle given by the sensor placed in $A$ is now $\theta^{*}$.

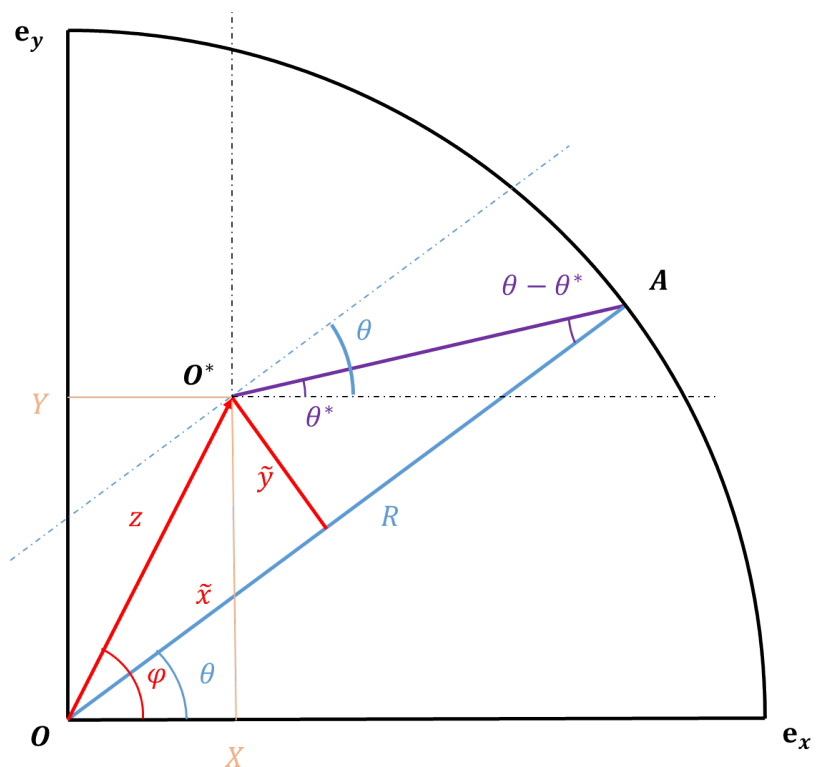

$$
\theta^{*} \simeq \theta-\arctan \left(\frac{\tilde{y}}{R}\right) \simeq \theta-\frac{\tilde{y}}{R}
$$

The angle is only impacted by the displacement in the normal direction to the sensor, which is coherent with the intuition. When 3 magnetic sensors are used, each one located at distance $R$ from $O$ at three different angles $\theta_{k}, k=1 \ldots 3$, then at any time $t \in \mathbb{R}$, the corresponding modified angle is thus written, using the same approximation,

$$
\begin{aligned}
\theta_{k}^{*}(t) & \simeq \theta_{k}+\frac{Z(t)}{R} \sin \left(\theta_{k}-\varphi(t)\right) \\
& =\theta_{k}+\frac{1}{R}\left(X(t) \sin \left(\theta_{k}\right)-Y(t) \cos \left(\theta_{k}\right)\right)
\end{aligned}
$$

where $X(t)=Z(t) \cos (\varphi(t))$ and $Y(t)=Z(t) \sin (\varphi(t))$.

When the shaft is rotating, each of the sensors gives a measurement $m_{k}(t)$, which is the angle of the shaft $\theta_{0}(t)$ with an offset corresponding to their angular position $\theta_{k}^{*}(t)$, 


$$
m_{k}(t)=\theta_{0}(t)+\theta_{k}^{*}(t) .
$$

A set of 3 equations is obtained and can be written in the matrix form

$$
\left[\begin{array}{l}
m_{1}(t) \\
m_{2}(t) \\
m_{3}(t)
\end{array}\right]=\left[\begin{array}{lll}
1 & \sin \left(\theta_{1}\right) & -\cos \left(\theta_{1}\right) \\
1 & \sin \left(\theta_{2}\right) & -\cos \left(\theta_{2}\right) \\
1 & \sin \left(\theta_{3}\right) & -\cos \left(\theta_{3}\right)
\end{array}\right]\left[\begin{array}{c}
\theta_{0}(t) \\
X(t) / R \\
Y(t) / R
\end{array}\right]+\left[\begin{array}{c}
\theta_{1} \\
\theta_{2} \\
\theta_{3}
\end{array}\right]
$$

or

$$
\mathbf{M}(t)=\mathbf{P V}(t)+\mathbf{\Theta} .
$$

When $\mathbf{P}$ is non-singular, i.e. when the three angles $\theta_{k}, k=1 \ldots 3$, are distinct, the displacements are obtained by inverting Eq.(16),

$$
\mathbf{V}(t)=\mathbf{P}^{-1}(\mathbf{M}(t)-\mathbf{\Theta}) .
$$

This last equation demonstrates that with 3 angle position sensors appropriately placed around the shaft, the orbit $(X(t), Y(t))$ can be estimated along with the angular position $\theta_{0}(t)$. In reality, each angle measurement is polluted by sources of strong noise, especially the geometric error due to the pole irregularities and the magnetic field distortion. A method to deal with these sources of errors is presented in section 4.5.

\subsection{Validation}
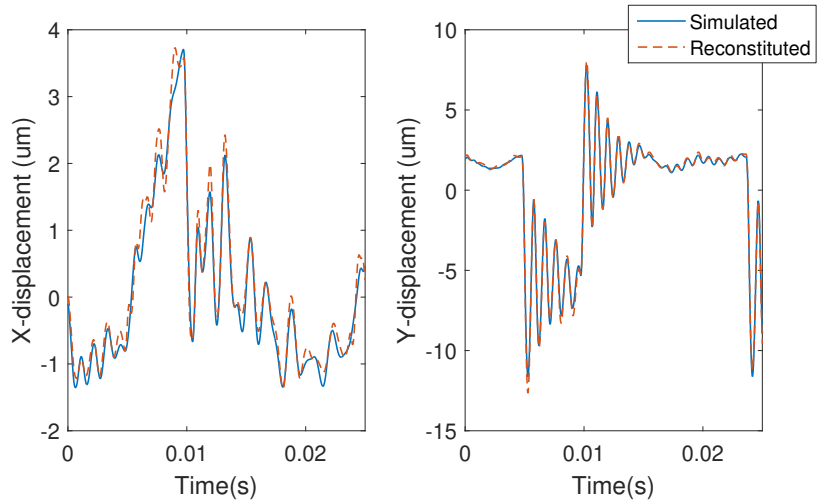

Figure 12: Magnetic simulation (in blue) and orbit reconstitution (in red) along horizontal (left) and vertical (right) axes respectively. 
A simulation has been conducted to validate the proposed method. The magnetic field produced by a 48 pole pairs encoder linked to the rotating shaft has been simulated using Finite Element Method and a magnetic field solver, the AMPERES commercial software. To obtain the magnetic field at arbitrary positions around the coder with sufficient accuracy, choice has been made to pre-compute the magnetic field in a neighbourhood of the encoder with a $1 \mu \mathrm{m}$ spatial resolution, and then to interpolate the resulting mesh thanks to cubic splines.

In this simulation, the sensor is not taken into account and thus the angle is computed directly from the components of an ideal magnetic field. Results are presented in Fig.12, where the orbit is computed from the angle position as proposed in Eq.(17), and the three angle measurements are retrieved from the orientation of the rotating magnetic field in the encoder tangent plane. As expected the reconstructed orbit matches the simulated one, with a deviation linked to the approximation done in Eq.(12).

\section{An experiment with three magnetic position sensors and prox- imity probes}

In order to validate the theoretical results obtained in the preceding sections, an experimental bench has been set up. The aim of the experiment is multiple:

1. Observe the shaft displacements in case of a bearing fault;

2. Demonstrate that the shaft orbit can be reconstituted solely from angle measurement sensors;

3. Show the relevance of the method to detect faulty bearings in the condition monitoring scope.

\subsection{Test rig}

For this purpose, a simple rotating shaft is monitored both with displacement and angle measurement sensors described in section 4.2. An endurance rig has been instrumented in order to reproduce natural bearing faults, and is illustrated in Fig.13. These faults can be obtained through accelerated test. The bearings and the configuration described in the simulation (see section 2.5, Fig.5 and Table 1) are used. The shaft is subjected to both axial and radial loads. 
Figure 13: Test bench used in the experiment. The main bearings (1) are 2 roller bearings SNR 22212EAW33. The test bearing (2) is a double row angular ball bearing SNR XTGB42000, with an magnetic tape used as angular reference. Radial load is applied through a spring system (3). The bench is an endurance bench used to reproduce natural bearing faults, such as the large defect presented in section 4.4 . 
A measurement campaign has been led, the rotation speed varying from $200 \mathrm{rpm}$ to $500 \mathrm{rpm}$. These conditions have been chosen in order to not deteriorate the faulty bearings when they are subjected to high loads.

\subsection{Sensors}

Proximity probes have been used to monitor the shaft displacements in presence of a bearing fault. Two sensors measuring the displacement in two orthogonal directions are used to completely describe the shaft trajectory in a radial plane. This approach is often favoured in the field of turbo-machinery where journal bearings are used [28], but it has not been investigated in the case of ball bearings. Some use cases include motor diagnosis [29] or large machinery monitoring, detecting macroscopic faults such as unbalance [30]. Dadouche et al. [31] explored the use of an eddy current sensor for bearing fault diagnosis, but used only one sensor and processed it as an accelerometer. Recently Zhong et al. [32] had the same approach with a different measurement device, using a camera coupled with a fringe pattern to observe the 3D-displacements of a rotating shaft.

In this experiment, the "ground truth" used as the shaft position reference is given by two contactless eddy current displacement sensors (ref. Kaman KD-2306). They are placed at a $90^{\circ}$ angle in order to retrieve the displacement of the shaft along 2 orthogonal axes, and thus the orbit in the rotation plane, as described in Fig.14.

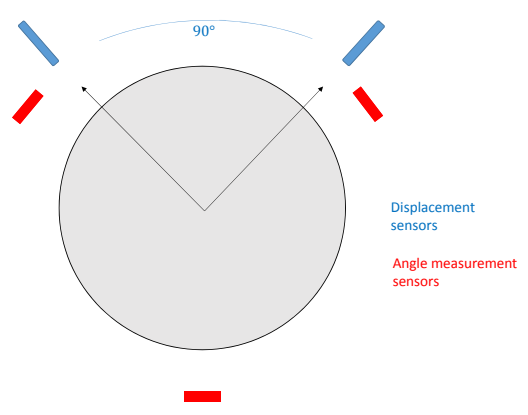

Figure 14: Sensor disposition in the experiment. The three angular sensors are placed at $120^{\circ}$ around the shaft, while the two displacement sensors are orthogonal to obtain the orbit in the radial plane. 
In parallel three angle position sensors are placed at $120^{\circ}$ one from each others all around the rotating shaft, as shown in Fig.14, such that the position matrix

$$
\mathbf{P}=\left[\begin{array}{ccc}
1 & 0 & -1 \\
1 & \frac{\sqrt{3}}{2} & \frac{1}{2} \\
1 & -\frac{\sqrt{3}}{2} & \frac{1}{2}
\end{array}\right]
$$

described in Eq.(16) is non-singular and well-conditioned. The three sensors are anisotropic magnetoresistances (AMR) coupled with a magnetic encoder placed around the shaft to output its angular position. NTN-SNR CPT63 sensors have been used in this experiment, the angle being computed from the magnetic field by the $\mathrm{iC}-H a u s s$ iC-MQ chipset at the sampling rate of $100 \mathrm{kHz}$. In order to obtain the orbit, Eq.(17) is used with the 3 angle sensor outputs.

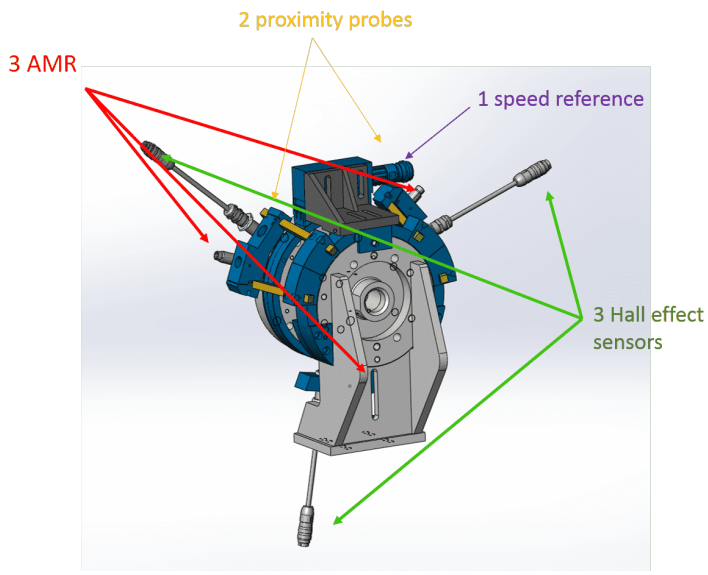

Figure 15: Experimental apparatus used to compare angle measurements done with AMR and displacements given by proximity probes. In this paper Hall effect sensors (in green) are not used.

\subsection{Acquisition system}

A custom acquisition card NI sbRIO-9627 is used to measure both digital and analog signals simultaneously. Two analog channels are used to sample the displacement signals given by the proximity probes at $50 \mathrm{kHz}$ with a resolution 16 bits, while three digital channels are used to sample the TTL signals coming from the angle measurement sensors at $80 \mathrm{MHz}$. A FPGA is used to compute the elapsed time between each edge of the sensors, following the common elapsed-time technique [9]. 


\begin{tabular}{|l|c|c|c|}
\hline Bearing & Origin of the defect & Width $(\mathrm{mm})$ & Area $\left(\mathrm{mm}^{2}\right)$ \\
\hline 1 & None (healthy) & 0 & 0 \\
2 & Artificial & 1.8 & 1 \\
3 & Artificial & 2.8 & 3.3 \\
4 & Natural & 6 & 13 \\
\hline
\end{tabular}

Table 2: List of defects.

\subsection{Faulty bearings}

The test bearing used during the experiment is an angular contact ball bearing described in Table 1. Localized defects, both artificial and natural, are present on the nonrotating inner ring of each tested bearing. A total of 4 bearings have been tested - three different defect sizes are studied, corresponding to small, medium and large size defects, along with an healthy bearing used as reference. They are illustrated in Fig.16. The size of each defect may be characterized by its width or its area as summarized in Table 2 ; in this work the depth of the defect has not been taken into account.
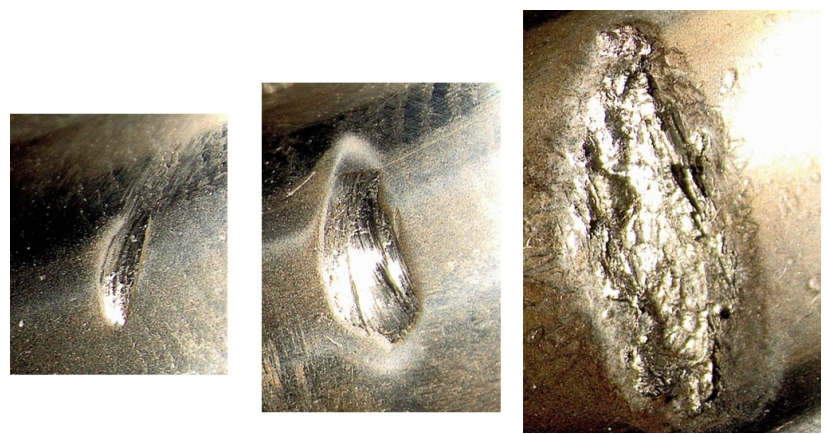

Figure 16: The three defects used in the experiment. The largest one, at right, is natural. The smaller ones, at left and centre, are artificial, as it has been difficult to obtain natural small size defects in this experiment.

\subsection{Comparison of measurements}

Both magnetic and eddy-current sensors measure the same phenomena from two different points of view. First, magnetic sensors measure the magnetic field emitted by the encoder fixed to the rotating shaft. Any displacement of the shaft results in a change in the magnetic field and thus in the measurement. When used as angle position sensors, part of the information 
is lost (only the direction of the magnetic field is measured) but as stated in section 3, this can be compensated by using several angle measurement sensors.

On the other hand, eddy-current sensors are influenced both by the distance to the shaft and the material conductivity. Thus the rotation of the shaft can only be observed indirectly, through eccentricity or conductivity variations in the shaft.

Because of these differences, the comparison of signals coming from both types of measurements is not obvious:

- The angle measurement is corrupted by the encoder signature, which is a combination of pole irregularities and distortion of the magnetic field. This signature, represented in Fig.17a, is a periodic pattern not present in the eddy current signals.

- Conductivity changes in the material impact the eddy current sensors with another periodic pattern (see Fig.17b), with the same periodicity (one revolution) but not present in the magnetic measurement.

For these reasons, signals are not directly compared; the study is focused on the displacement component induced by the bearing fault, which is decoupled from other phenomena. Indeed, the period between two rolling element crossings through the defective zone is not synchronous with the shaft revolution, which can be used advantageously, so that the displacement linked to the bearing fault can be isolated from other sources, and compared in both measurements.

\subsection{Isolating the defect linked component}

The slight modifications of the orbit induced by a localized defect are challenging to observe, because they are hidden by macroscopic variations such as eccentricity of the shaft (see Fig.17b). These phenomena are better described in the angular domain, as they are linked to the rotation of the shaft. Moreover, angular domain is adapted to the study of faulty bearings, as the orbit will be disturbed by the defect each time a rolling element goes through the flaking zone - i.e. at a fixed angular interval. Two steps are necessary to isolate the defect linked component in the displacement signals:

- First, both displacement signals are resampled using the angle position sensor as a reference. The resulting signals are then in the order domain. 


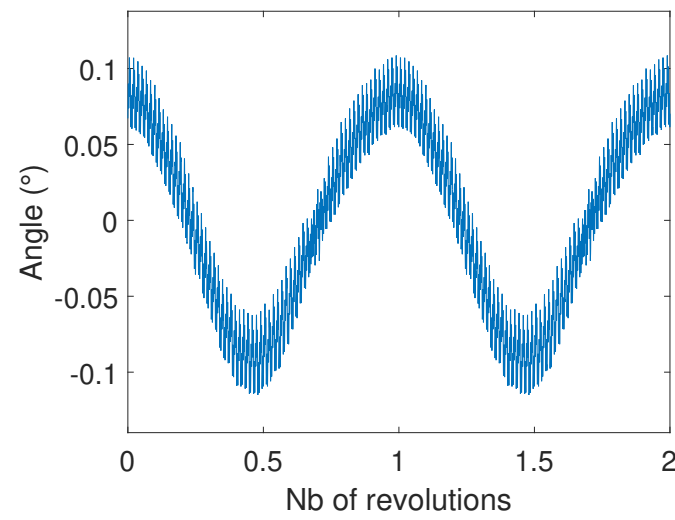

(a) Periodic pattern observed on the angle measurement sensors. For visualization purposes, the represented signal is the difference between the actual angle measurement and a linearly increasing angle obtained at an ideal constant speed. High frequency oscillations are due to distortion of the magnetic field for each pole.

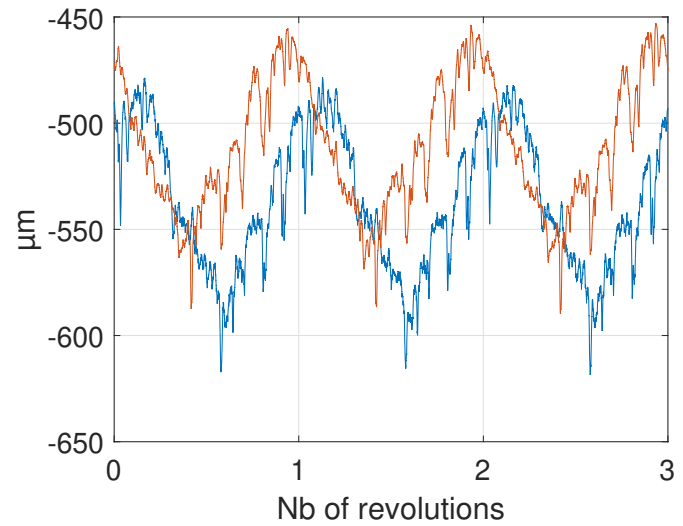

(b) Signals obtained from the two displacement sensors. A periodic pattern linked to the shaft revolution is observed, due to both conductivity changes and eccentricity of the shaft. The $90^{\circ}$ phase shift between sensors is clearly observed as a delay between sensor 1 (in blue)) and sensor 2 (in red)) outputs.

Figure 17: Observations of sensors signatures in each kind of measurements. These phenomena are dominant in the signals and cannot be compared, coming from different physical origins.

- Then angular synchronous average is performed to remove the revolutionperiodic component and isolate the defect-linked component

Two strategies are followed successively to perform synchronous average: the first one averages the signal at the rotation frequency to estimate both the periodic pattern (in the angle measurement case) and the macroscopic component (in the displacement measurement case), which are removed. Then residues are averaged a second time, directly at the fault frequency, to isolate the component of interest from other components. In this case, the inner ring characteristic frequency is estimated from the bearing geometry and is theoretically equal to $f_{0}=6.65$ orders. In practice, the fault frequency is not known with accuracy and this latter operation is not straightforward: both resampling errors and slippage inside the bearing lead to spectral smearing, so that the synchronous average is not able to retrieve the defect-linked component.

This phenomena may be explained from a frequency perspective: as described by Braun [33], the synchronous average may be viewed as a comb filter at the frequency corresponding to the synchronous period, and all its 
harmonics. The bandwidth of the filter is inversely proportional to the number of averaged cycles, so that averaging a large number of cycles leads to very narrow filters. As a result, any smearing in the spectrum, or any error in the estimation of the synchronous frequency, may lead to catastrophic results.

To avoid these problems, a two-step strategy has been followed here:

1. First the exact fault frequency $\hat{f}_{0}$ is estimated from the spectrum $X(f)$, using the maximum amplitude in a given search range $\delta_{f}$ around the theoretical value $f_{0}$

$$
\hat{f}_{0}=\underset{f \in\left[f_{0}-\delta_{f}, f_{0}+\delta_{f}\right]}{\arg \max }|X(f)|^{2}
$$

Note that better precision is achieved using a high harmonic, say the $k^{\text {th }}$ harmonic $f_{k}$ of the fault frequency, and estimating $\hat{f}_{0}$ as

$$
\hat{f}_{0}=\frac{\hat{f}_{k}}{k}
$$

2. Use the cage frequency as the synchronous frequency, defined as

$$
F T F=\frac{\hat{f}_{0}}{N_{b}}
$$

where $N_{b}$ is the number of rolling elements in the bearing (here $N_{b}=$ 11). This reduces the number of averages done so that the orbit of each rolling element may be observed independently.

Results are illustrated in Fig.18.

\subsection{Denoising signals}

In this experiment, an other source of noise must be taken into account, a resonance effect polluting the displacement signals. This effect can be clearly observed when measurements are done at different speeds. Hence the resonance is observed in a specific frequency band, which does not depend on the rotation speed. However, after angular resampling, increasing the rotation speed can be viewed as increasing the angular sampling rate, and thus it moves the original resonance towards low orders. An illustration is given Fig.19.

To be able to compare signals at different speeds, the resonance effect is cancelled using a notch filter in the corrupted frequency band. 


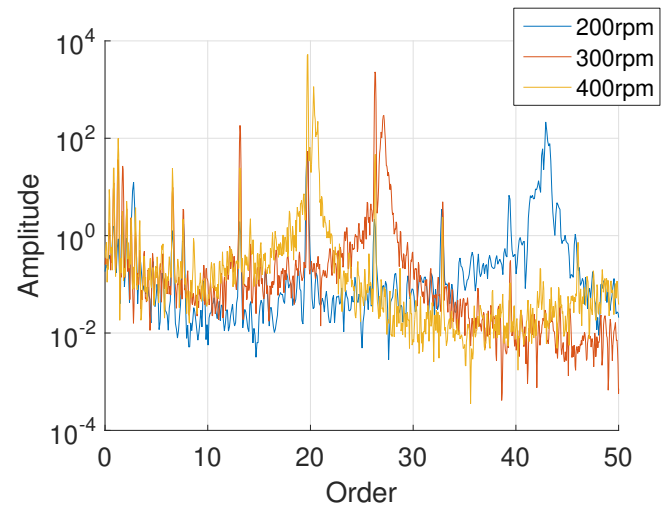

(a) Resonance observed in the PSD of resampled displacement signals. When speed increases, the resonance peak moves towards low orders.

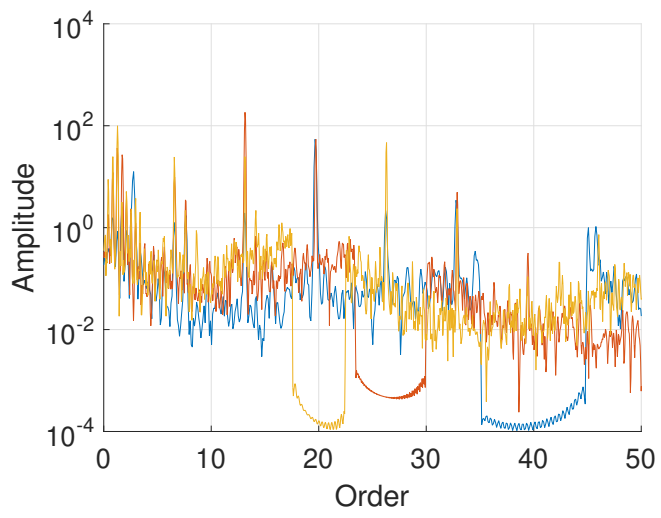

(b) A notch filter is used to remove the resonance so that peaks linked to the fault are now dominant in the spectrum.

Figure 19: Observation and removal of the resonance at three different rotation speeds. Displacement signals have been resampled with the angular reference and the Power Spectrum Density has been computed with Welch method. 


\subsection{Results}

After these steps, two signals representative of the shaft displacement in two orthogonal directions are used to plot the orbit in a 2D plane. Equivalently, three angle measurement signals are combined to reconstruct the shaft orbit. Fig.20 shows the orbits obtained for these two types of signals in the healthy case and with the largest defect. The first observation is that there is a significant difference between the behaviour of the shaft in both situations: in case of an healthy bearing, the amplitude of the orbit is about $1 \mu \mathrm{m}$, while it is multiplied by a factor 10 in the case of the largest defect. The second observation is that this information may be retrieved both with orbits obtained by direct measurement (the proximity probes) and with reconstructed orbits obtained by the presented method (the angle sensors). A third point is that the vertical direction is favoured in the trajectories obtained in the defective case: this coincides with the vertical direction in which the defect has been oriented, and confirms that the shaft is moving each time a rolling element is unloaded when entering the fault zone.

To confirm this last observation, displacement signals have been projected onto the maximum variance direction using a Principal Component Analysis to perform further analysis. More precisely, let $\left(x_{n}, y_{n}\right)_{n \in 1 . . N}$ be the couples of sampled points given by both displacement sensors, and $X=\left(\begin{array}{cc}x_{1} & y_{1} \\ \vdots & \vdots \\ x_{N} & y_{N}\end{array}\right)$.

The covariance matrix is given by

$$
R=\frac{1}{N} X^{T} X
$$

The eigenvector $V$ associated with the maximum eigenvalue $\lambda$ of $R$ is used to project the trajectory onto the maximum variance direction, giving the new signal

$$
Z=X V
$$

The obtained direction is almost vertical and corresponds to the fault location, showing that the shaft trajectory is disturbed when rolling elements pass through the defective zone. In Fig.21, this event is observed as a shaft displacement at the fault characteristic frequency, i.e. 6.65 times per revolution. The shaft oscillation has a rectangular shape, reflecting both entry and 
exit of the rolling element in the defect zone, and the displacement amplitude being coherent with the simulation.
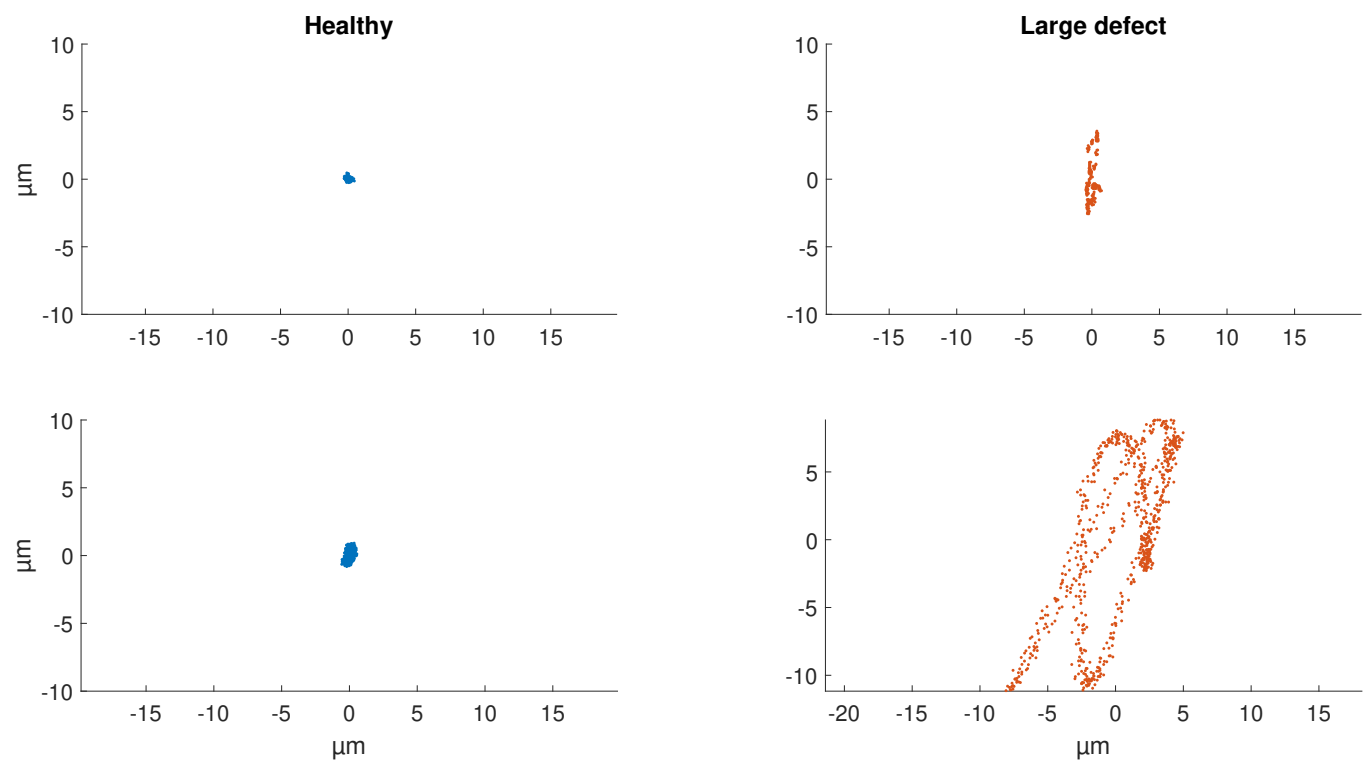

Figure 20: Illustration of the orbits obtained both without defect (left) and with defect (right); the top row shows orbits obtained with eddy-current sensors, the bottom one shows reconstructed orbits obtained with angular sensors.

A direct consequence is that care should be taken when using angle measurement sensors to monitor a rotating machine through IAS signals. As suggested by Eq.(11), the vertical displacement observed here would not be measured by a sensor placed vertically. It can be deduced that an optimal position may exist, but without prior information this position remains unknown.

Amplitude differences can be noticed when comparing the two different approaches, and several hypotheses may explain them:

1. The mechanical apparatus used to position the eddy-current sensors is subject to vibrations, thus the signals have been processed to remove a masking resonance effect thanks to a narrow notch filter, as explained in section 4.7. It appears that this resonance does not appear in the angle measurements, which are fixed to a distinct part of the machine.

2. Due to mechanical constraints, both types of sensors could not be placed at the same exact point on the shaft. Vibration nodes could then alter the results. 


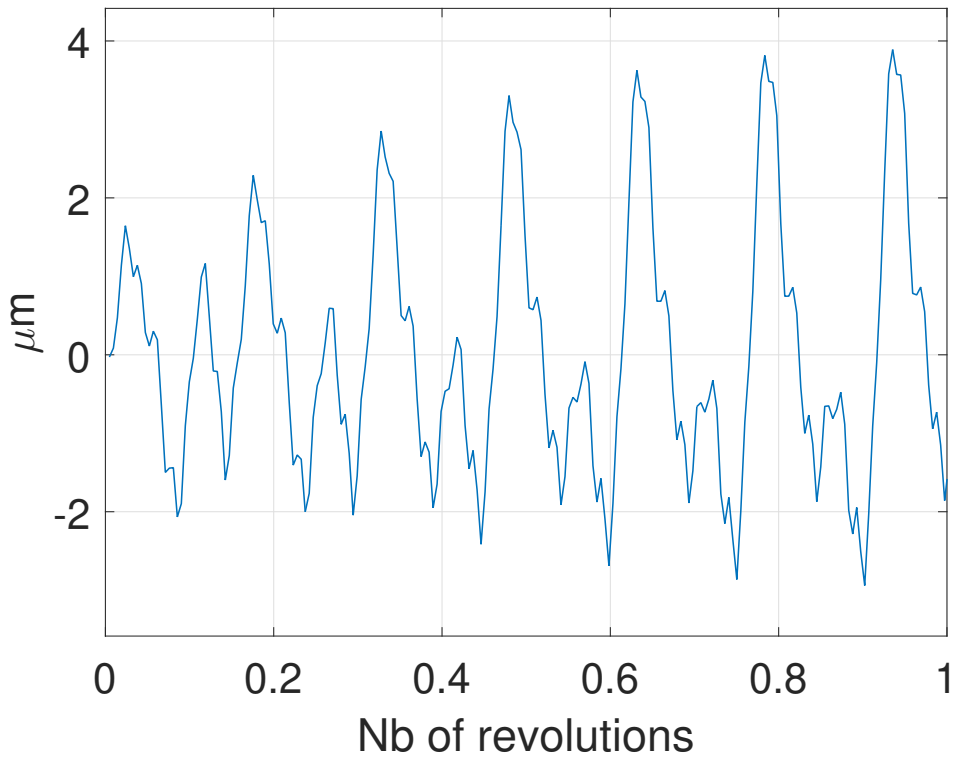

Figure 21: Displacement obtained after projection of displacement measurements on the most energetic direction, large defect, 500rpm. A displacement is observed each time a rolling element passes through the defective zone, 6.65 times per revolution.

Improvements might be obtained using a more compact apparatus, making sure that proximity probes do no vibrate to avoid the destructive filtering operation described previously.

Following these encouraging observations, the next section gives detailed results obtained with the full dataset in order to valid the assumptions.

\subsection{Fault severity indicator}

In order to characterize each failure case, a severity indicator is derived from these experimental signals. A very direct approach is to compute the energy of the projected signal, energy given by the maximum eigenvalue $\lambda$ of the covariance matrix $R$. Thus $\lambda$ can be used as a fault severity indicator, but a more convenient one is obtained remarking that eigenvalues of the covariance matrix can be viewed as the axes of the ellipse underlying the data points in the least square sense [34]. Hence the semi-major axis can be computed as $L=\sqrt{2 \lambda}$, given in micrometers in Fig. 22 .

These experimental results confirm the conclusions obtained from the mechanical model. They show that shaft displacement measurements provide useful information for characterizing a significant defect, despite some fluc- 

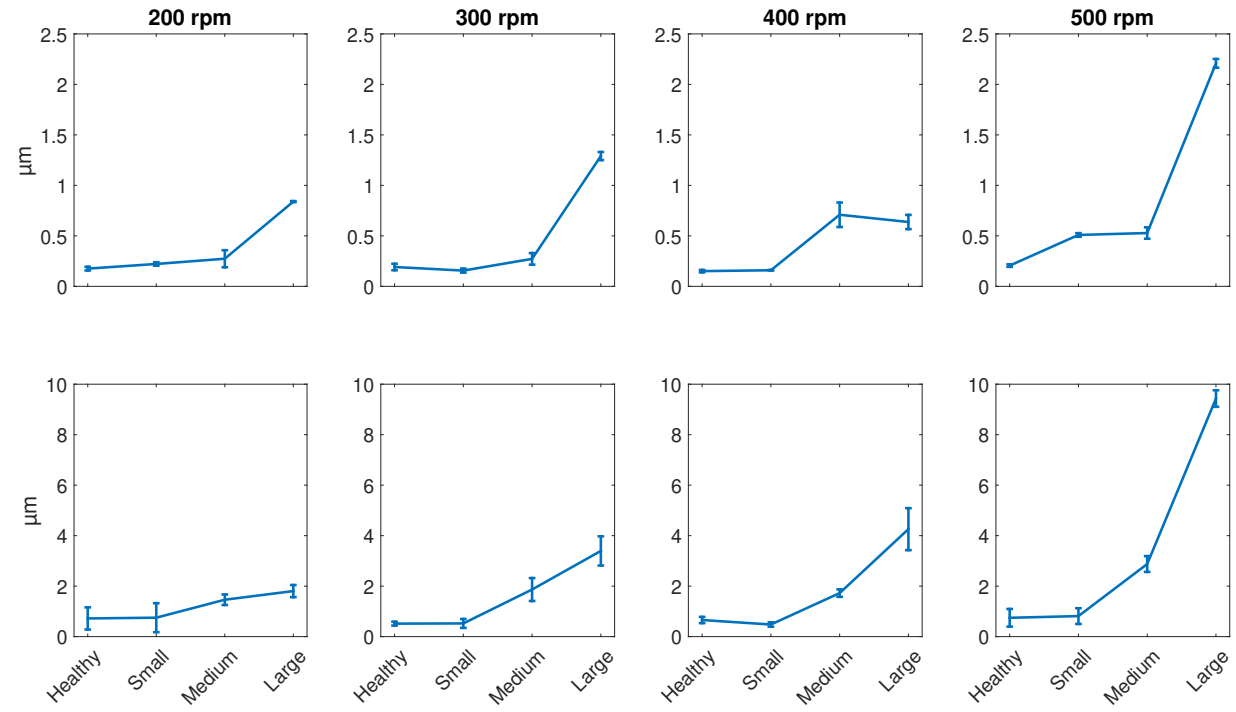

Figure 22: Indicator obtained from the orbit ellipse semi-major axis, computed from the maximum eigenvalue of the covariance matrix between the two orthogonal signals. Top row shows results obtained with eddy-current sensors, the bottom one shows those obtained with angle sensors. While good results are obtained for the two largest defects, the small defect is not separated from the healthy case.

tuations of the indicator as signals have been heavily filtered to counteract the effects of various noise source.

As the smallest defect cannot be detected directly with the proposed approach, it is worth mentioning that a much more reliable indicator can be derived in the frequency domain. In this example a simple energetic criterion is adopted, based on the energy computed at the fault characteristic frequency and its harmonics. It is computed directly from the Power Spectral Density obtained by Welch method, taking into account the asymmetry of the spectrum when dealing with complex signals that are used to represent the two-dimensional orbits. Fig.23 and Fig.24 represent the mean energy obtained from both positive and negative frequencies associated to each harmonic taken separately, using displacement and angle sensors respectively. It can be seen that for most harmonics, the energy is increasing with the defect size, independently of the rotation speed (given in colours). When dealing with displacement sensors, the main information is provided by the first five harmonics, while the energy is spread all along the first ten harmonics in the reconstructed orbits. As the objective is to separate the 4 different bearings, 
it is noticed that the first harmonic gives no information, at least from this energetic point of view, and should be discarded in order to improve the classification results.

Fig. 25 presents a global indicator obtained by summing the contribution of each harmonic, showing that each defect can be properly classified, and that this time the method achieves good discrimination between the small defect and the healthy bearing.

More sophisticated classification methods may eventually achieve better performances and will be discussed in future works, as the objective of this paper as been fulfilled: orbits have been proven to carry useful information about the degradation state of the bearing, thus underlying the proposed approach based on the angular reconstruction of the shaft displacements.
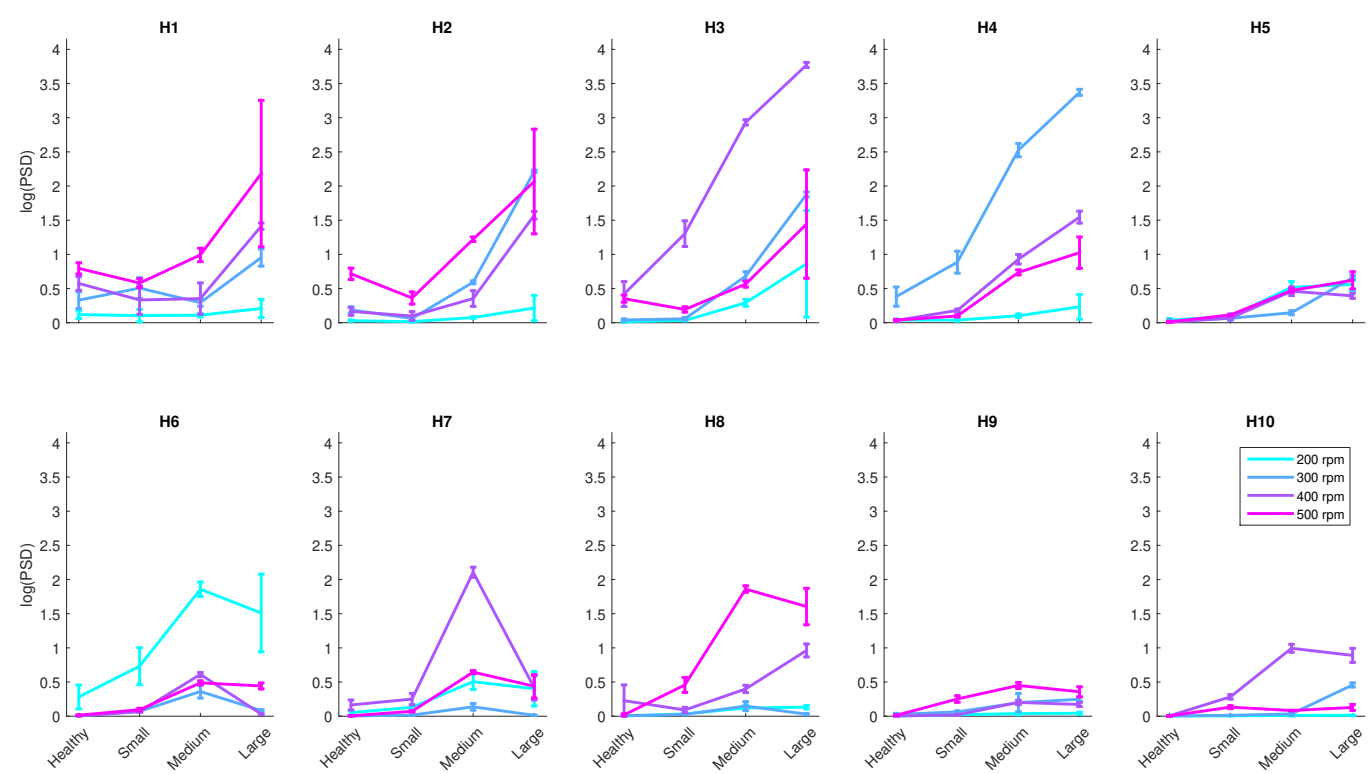

Figure 23: Energy observed at the 6.65 fault frequency and its 10 first harmonics, for 4 different speeds, computed from displacement sensors. Vertical bars display the standard deviation obtained for 5 measurements. Most of the energy is localized on the first few harmonics. 
H1

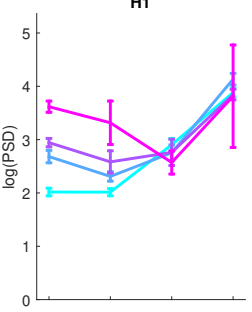

H6

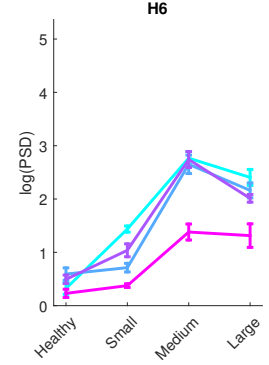

H2

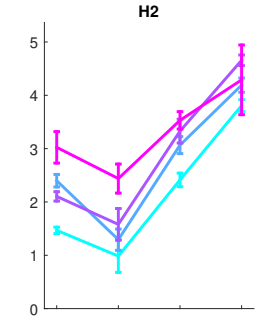

H7

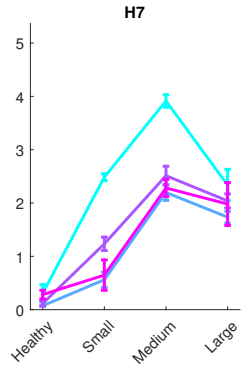

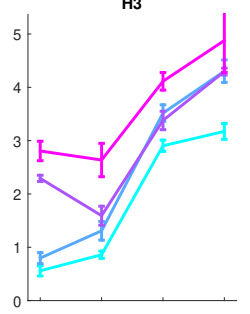

H8

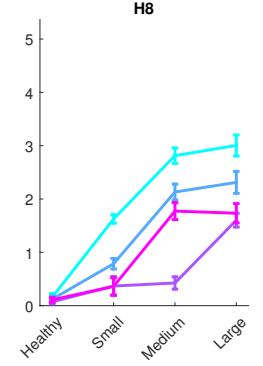

H4

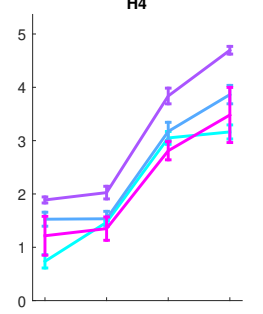

H9

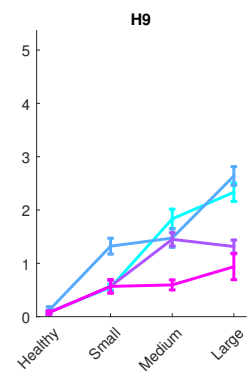

H5
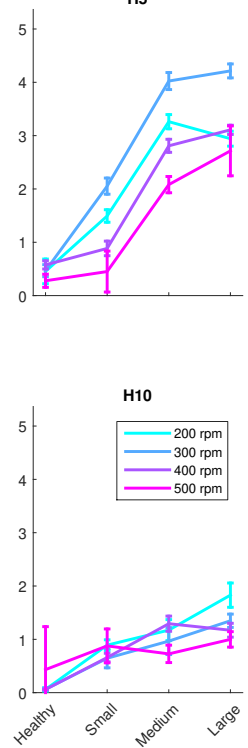

Figure 24: Energy observed at the 6.65 fault frequency and its 10 first harmonics computed from angle sensors.. Vertical bars display the standard deviation obtained for 5 measurements. Energy is well spread along all the harmonics, but the fundamental (H1) shows bad behaviour and has been discarded in the indicator computation done in Fig.25.
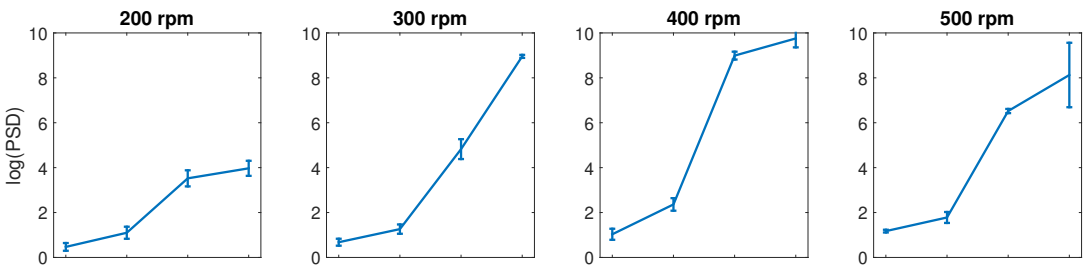


\section{Conclusion}

In this paper, a new approach to condition monitoring of rotating machines has been presented. A mechanical model has been proposed to describe a bearing fault, and the contact forces have been derived in the damaged zone in an original way. Both simulations and experiments were conducted to show that a bearing fault signature is observable in the shaft orbit. A theoretical model has been developed to show that angle measurement sensors are sensitive to shaft displacements, so that a set of at least three sensors can be used to retrieve this information. Based on this original principle, an experiment was conducted where different bearing faults cases have been studied. An indicator based on the orbit shape has been derived. Results showed on one hand that the method achieves correct orbit reconstruction and on the other hand that it can be used for the diagnosis of faulty bearings. One advantage of this method is the possibility to use the angular reference to work in angular domain, which is very convenient for the analysis of rotating machine to get rid of speed variations. In further research, different magnetic technologies will be tested to improve the sensitivity of the sensors and thus achieve better discrimination results. Looking at the numerous advantages that simple angular magnetic sensors bring, the proposed method is already an appealing alternative to more classical sensors such as vibration or acoustic ones.

\section{Appendix}

Let $\gamma: \mathbb{R} \rightarrow \mathbb{R}$ be a function of class $C^{1}$. Each couple $(\theta, \gamma(\theta))$ is used to describe the curve defined by the bearing raceway. The direction of the tangential vector to the curve is given by $\dot{\gamma}(\theta)$ such that for any vector $T$ tangential to the curve at the point $(\theta, \gamma(\theta))$,

$$
\frac{\left\langle T, e_{r}\right\rangle}{\left\langle T, e_{\theta}\right\rangle}=\dot{\gamma}(\theta)
$$

Then for a normal vector $N$ to the curve at the same point, with $\langle T, N\rangle=$ 0 the inverse relationship is

$$
\frac{\left\langle N, e_{\theta}\right\rangle}{\left\langle N, e_{r}\right\rangle}=-\dot{\gamma}(\theta) .
$$


It leads to

$$
\|N\|^{2}=\left\langle N, e_{r}\right\rangle^{2}+\left\langle N, e_{\theta}\right\rangle^{2}=\left\langle N, e_{r}\right\rangle^{2}\left(1+\dot{\gamma}(\theta)^{2}\right)
$$

and finally

$$
\left\langle N, e_{r}\right\rangle= \pm\|N\|\left(1+\dot{\gamma}(\theta)^{2}\right)^{-1 / 2},
$$

the sign being determined by the orientation of the vector.

\section{References}

[1] J. Mathew and R. J. Alfredson. The condition monitoring of rolling element bearings using vibration analysis. Journal of vibration, acoustics, stress, and reliability in design, 106(3):447-453, 1984.

[2] R. B. Randall. Vibration-based condition monitoring: industrial, aerospace and automotive applications. John Wiley \& Sons, 2011.

[3] D. Mba and Raj B. K. N. Rao. Development of acoustic emission technology for condition monitoring and diagnosis of rotating machines; bearings, pumps, gearboxes, engines and rotating structures. The Shock and Vibration Digest, 38(1):3-16, 2006.

[4] G. E. Newell. Oil analysis cost-effective machine condition monitoring technique. Industrial Lubrication and Tribology, 51(3):119-124, 1999.

[5] F. Rafieian, F. Girardin, Z. Liu, M. Thomas, and B. Hazel. Angular analysis of the cyclic impacting oscillations in a robotic grinding process. Mechanical Systems and Signal Processing, 44(1-2):160-176, 2014. ISSN 0888-3270. doi: 10.1016/j.ymssp.2013.05.005. Special Issue on Instantaneous Angular Speed (IAS) Processing and Angular Applications.

[6] M. Ritou, S. Garnier, B. Furet, and J. Y. Hascoet. Angular approach combined to mechanical model for tool breakage detection by eddy current sensors. Mechanical Systems and Signal Processing, 44(1-2):211220, 2014. ISSN 0888-3270. doi: 10.1016/j.ymssp.2013.02.004. Special Issue on Instantaneous Angular Speed (IAS) Processing and Angular Applications. 
[7] M. Lamraoui, M. Thomas, M. El Badaoui, and F. Girardin. Indicators for monitoring chatter in milling based on instantaneous angular speeds. Mechanical Systems and Signal Processing, 44(1-2):72-85, 2014. ISSN 0888-3270. doi: 10.1016/j.ymssp.2013.05.002. Special Issue on Instantaneous Angular Speed (IAS) Processing and Angular Applications.

[8] L. Renaudin, F. Bonnardot, O. Musy, J. B. Doray, and D. Rémond. Natural roller bearing fault detection by angular measurement of true instantaneous angular speed. Mechanical Systems and Signal Processing, 24(7):1998-2011, 2010. ISSN 0888-3270. doi: 10.1016/j.ymssp.2010.05. 005. Special Issue: ISMA 2010.

[9] Yuhua Li, Fengshou Gu, Georgina Harris, Andrew Ball, Nick Bennett, and Ken Travis. The measurement of instantaneous angular speed. $M e-$ chanical Systems and Signal Processing, 19(4):786-805, 2005.

[10] M. El Badaoui and F. Bonnardot. Impact of the non-uniform angular sampling on mechanical signals. Mechanical Systems and Signal Processing, 44(1-2):199-210, 2014. ISSN 0888-3270. doi: 10.1016/j.ymssp.2013. 10.008. Special Issue on Instantaneous Angular Speed (IAS) Processing and Angular Applications.

[11] H. André, F. Girardin, A. Bourdon, J. Antoni, and D. Rémond. Precision of the ias monitoring system based on the elapsed time method in the spectral domain. Mechanical Systems and Signal Processing, 44 (1-2):14-30, 2014. ISSN 0888-3270. doi: 10.1016/j.ymssp.2013.06.020. Special Issue on Instantaneous Angular Speed (IAS) Processing and Angular Applications.

[12] Q. Leclere, F. Girardin, and D. Rémond. An analysis of instantaneous angular speed measurement errors. Proceedings of Surveilliance, 7, 2013.

[13] A. Palermo, K. Janssens, and L. Britte. Evaluation and improvement of accuracy in the instantaneous angular speed (ias) and torsional vibration measurement using zebra tapes. In Advances in Condition Monitoring of Machinery in Non-Stationary Operations, volume 4 of Applied Condition Monitoring, pages 43-56. Springer International Publishing, 2016. ISBN 978-3-319-20462-8. doi: 10.1007/978-3-319-20463-5_4. 
[14] A. Bourdon, H. André, and D. Rémond. Introducing angularly periodic disturbances in dynamic models of rotating systems under nonstationary conditions. Mechanical Systems and Signal Processing, 44 (1-2):60-71, 2014. ISSN 0888-3270. doi: 10.1016/j.ymssp.2013.09.004. Special Issue on Instantaneous Angular Speed (IAS) Processing and Angular Applications.

[15] A. Bourdon, S. Chesné, H. André, and D. Rémond. Reconstruction of angular speed variations in the angular domain to diagnose and quantify taper roller bearing outer race fault. Mechanical Systems and Signal Processing, 120:1-15, 2019. ISSN 0888-3270. doi: 10.1016/j.ymssp.2018. 09.040.

[16] J. L. Gomez, A. Bourdon, H. André, and D. Rémond. Modelling deep groove ball bearing localized defects inducing instantaneous angular speed variations. Tribology International, 2016.

[17] N. Tandon and A. Choudhury. An analytical model for the prediction of the vibration response of rolling element bearings due to a localized defect. Journal of Sound and Vibration, 205(3):275-292, 1997.

[18] J. Sopanen and A. Mikkola. Dynamic model of a deep-groove ball bearing including localized and distributed defects, part 2: Implementation and results. Proc. Instn. Mech. Engrs., Part K: Journal of Multi-Body Dynamics 217, pages 213-223, 2003.

[19] N. Sawalhi and R. B. Randall. Simulating gear and bearing interactions in the presence of faults part. i. the combined gear bearing dynamic model and simulation of localized bearing faults. Mechanical Systems and Signal Processing, 22:1924-1951, 2008.

[20] D. S. Shah and V. N. Patel. A review of dynamic modeling and fault identifications methods for rolling element bearing. Procedia Technology, 14:447-456, 2014. ISSN 2212-0173. doi: 10.1016/j.protcy.2014.08.057. 2nd International Conference on Innovations in Automation and Mechatronics Engineering, ICIAME 2014.

[21] N. Sawalhi and R. B. Randall. Vibration response of spalled rolling element bearings: Observations, simulations and signal processing techniques to track the spall size. Mechanical Systems and Signal Processing, 25(3):846-870, 2011. 
[22] Jing Liu, Yimin Shao, and W. D. Zhu. A new model for the relationship between vibration characteristics caused by the time-varying contact stiffness of a deep groove ball bearing and defect sizes. Journal of Tribology, 137(3):031101, 2015.

[23] C. Mishra, A. K. Samantaray, and G. Chakraborty. Ball bearing defect models: A study of simulated and experimental fault signatures. Journal of Sound and Vibration, 400:86-112, 2017.

[24] Y. W. Kwon and H. Bang. The finite element method using MATLAB. CRC press, 2000.

[25] H. Panzer, J. Hubele, R. Eid, and B. Lohmann. Generating a parametric finite element model of a $3 \mathrm{~d}$ cantilever timoshenko beam using matlab. Tech. reports on aut. control, Inst. Aut. Control, TU München, 2009.

[26] T. A. Harris. Rolling bearing analysis. John Wiley and sons, 2001.

[27] L. F. Shampine and M. W. Reichelt. The matlab ode suite. SIAM journal on scientific computing, 18(1):1-22, 1997.

[28] N. Bachschmid, P. Pennacchi, and A. Vania. Identification of multiple faults in rotor systems. Journal of Sound and Vibration, 254(2):327-366, 2002. ISSN 0022-460X. doi: 10.1006/jsvi.2001.4116.

[29] J. Urresty, R. Atashkhooei, J. Riba, L. Romeral, and S. Royo. Shaft trajectory analysis in a partially demagnetized permanent-magnet synchronous motor. IEEE Transactions on Industrial Electronics, 60(8): 3454-3461, 2013.

[30] D. F. Shi, W. J. Wang, P. J. Unsworth, and L. S. Qu. Purification and feature extraction of shaft orbits for diagnosing large rotating machinery. Journal of Sound and Vibration, 279(3-5):581-600, 2005.

[31] A. Dadouche, A. Rezaei, V. Wickramasinghe, W. Dmochowski, J. W. Bird, and F. Nitzsche. Sensitivity of air-coupled ultrasound and eddy current sensors to bearing fault detection. Tribology Transactions, 51 (3):310-323, 2008. 
[32] Jianfeng Zhong, Shuncong Zhong, Qiukun Zhang, Shulin Liu, Zhike Peng, and Nuno Maia. Real-time three-dimensional vibration monitoring of rotating shafts using constant-density sinusoidal fringe pattern as tri-axial sensor. Mechanical Systems and Signal Processing, 115:132-146, 2019. ISSN 0888-3270. doi: 10.1016/j.ymssp.2018.05.049.

[33] S. Braun. The synchronous (time domain) average revisited. Mechanical Systems and Signal Processing, 25(4):1087-1102, May 2011. ISSN 08883270. doi: 10.1016/j.ymssp.2010.07.016.

[34] A. Fitzgibbon, M. Pilu, and R. B. Fisher. Direct least square fitting of ellipses. IEEE Transactions on pattern analysis and machine intelligence, 21(5):476-480, 1999. 